\title{
The global challenge of Candida auris in the intensive care unit
}

\author{
Andrea Cortegiani $i^{*} \mathbb{D}$, Giovanni Misseri', Antonino Giarratano ${ }^{1}$, Matteo Bassetti ${ }^{2}$ and David Eyre ${ }^{3}$
}

Since the first isolation of Candida auris in 2009, scientific community has witnessed an exponential emergence of infection episodes and outbreaks in different world regions [1]. According to the Centers for Disease Control and Prevention (CDC), 560 cases of $C$. auris infections have been notified in the United States as 31 January 2019. It is likely that many cases are missed, due to its misidentification with other non-albicans Candida spp. (e.g., C. haemulonii) by common microbiological diagnostic methods (https://www.cdc.gov/fungal/diseases/candidiasis/tracking-c -auris.html). Most of the reports occurred in critically ill adults, with risk factors for invasive fungal infections, such as immunosuppression, surgery, or indwelling catheters. The most common form of infection was candidemia, with a crude mortality of nearly $30 \%$, but up to $70 \%$ in some reports [2].

Despite implementation of countermeasures to limit colonization and infections in intensive care units (ICUs), cases continue to be reported, with a tendency to an endemic pattern [3]. This reflects the ability of $C$. auris to persist in clinical environment, facilitating its transmission within critical care setting. Multidrug-resistant (MDR) pattern and has been frequently observed (around 40\%) with serious and complex consequences for antifungal therapy [4].

In view of $C$. auris progressive spread and treatment concerns, attention should be focused on the following A.U.R.I.S. major issues (Fig. 1):

1. Worldwide Alert

2. AntifUngal treatment resistance

3. Resilience and mechanisms of transmission

4. Implementation of infection prevention and control measures

5. Surveillance

\footnotetext{
* Correspondence: andrea.cortegiani@unipa.it

${ }^{1}$ Department of Surgical, Oncological and Oral Science (Di.Chir.On.S.). Section of Anesthesia, Analgesia, Intensive Care and Emergency. Policlinico Paolo Giaccone, University of Palermo, Via del vespro 129, 90127 Palermo, Italy Full list of author information is available at the end of the article
}

\section{Worldwide Alert}

Following the first isolation in Japan, cases have been reported in several countries in five continents. Although uncommon for fungi, $C$. auris has the ability to cause outbreaks, as seen in India, the UK, Spain, the USA, Venezuela, Colombia, and South Africa [1]. It is still debated whether $C$. auris emerged in one region with subsequent spreading to others, or if it emerged independently across different countries. Evidence from genomic sequencing demonstrates different clades of $C$. auris show strong geographic structure, with independent emergence in East and South Asia, Africa, and South America $[1,5,6]$.

\section{AntifUngal treatment resistance}

To date, there are not established minimum inhibitory concentrations (MICs) breakpoints for susceptibility testing of $C$. auris. Antifungal susceptibility data from three continents demonstrated that nearly $40 \%$ were MDR, with strains being resistant to fluconazole (90\%), amphotericin B (30-40\%) and echinocandins (5-10\%). Moreover, a small percentage were also resistant to all antifungals actually available $[4,6]$. C. auris demonstrates a high propensity to develop antifungal resistance under selective pressure. Recent studies demonstrated mutations in ERG11 (encoding lanosterol demethylase, the target of azoles) and FKS1 genes (encoding 1,3-beta-glucan synthase, the target of echinocandins) [1, 7].

The recommended antifungals for $C$. auris treatment are mainly based on in vitro testing and on the most frequently retrieved resistance profiles. Echinocandins are the recommended first-line treatment, pending specific susceptibility testing. Lipid formulation of amphotericin B should be an alternative in patients not responding to echinocandins. Close monitoring to early detect therapeutic failures and evolution of antifungal resistance is needed. New antifungals (e.g., SCY-078, APX001A/APX001, and rezafungin) have been tested with success but they are not available to date for clinical use [1]. 


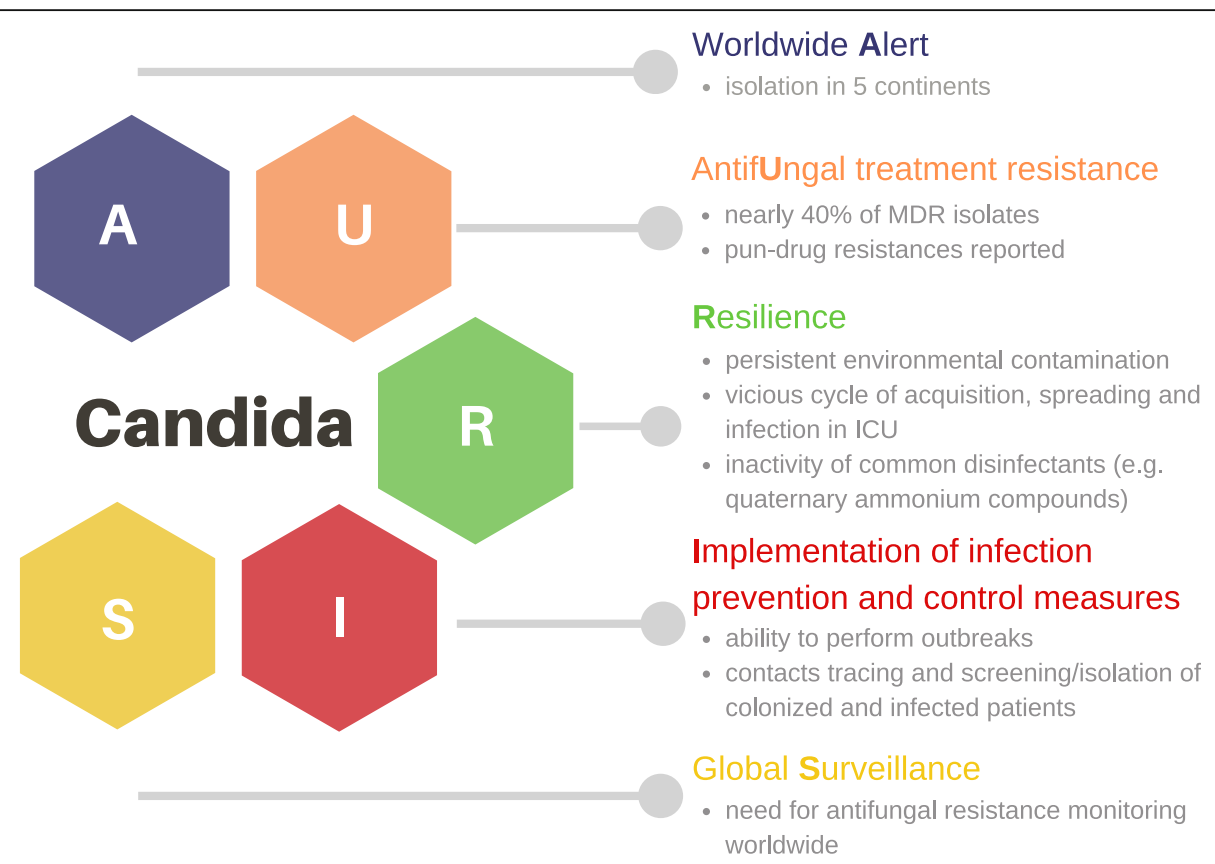

Fig. 1 Major issues related to Candida auris. Major issues related to Candida auris described with A.U.R.I.S. outline. MDR, multidrug resistant; ICU, intensive care unit

\section{Resilience and mechanisms of transmission}

Unlike others Candida species, C. auris can colonize different anatomical sites (e.g., skin, skin, rectum, axilla, stool) and contaminate hospital equipment and surfaces, creating a vicious cycle of acquisition, spreading, and infection, particularly in ICUs. Indeed, bed, chairs, and monitoring tools (e.g., pulse oximeters, temperature probes) were contaminated during outbreaks [8]. Recently, Eyre et al. [9] published the results of a patients' and hospital environmental screening program in Oxford, UK, after 70 patients (66 admitted to a neuro-ICU) were identified as being colonized or infected by $C$. auris. Seven patients developed an invasive infection during hospital stay. C. auris was detected mainly on skin-surface axillary temperature probes and other reusable tools. In patients monitored with skin-surface temperature probes, the risk of $C$. auris infection/colonization was seven times higher. Adoption of specific bundles of infection control had no significant effects until removal of the temperature probes [9].

Recent studies have confirmed that $C$. auris can form biofilms, with a high variation of capacity of production depending on the $C$. auris strain considered [10]. Biofilm may present reduced susceptibility to hydrogen peroxide and chlorhexidine [11].

Quaternary ammonium compounds and cationic surfaceactive products seem to be ineffective against $C$. auris. Chlorine-based products appear to be the most effective for environmental surface disinfection [12]. Chlorine-based disinfectants (at a concentration of $1000 \mathrm{ppm}$ ), hydrogenperoxide, or other disinfectants with documented fungicidal activity are recommended for environmental cleaning by the European CDC (ECDC) [13].

\section{Implementation of infection prevention and control measures}

CDC and ECDC released recommendations for $C$. auris case and outbreak management [13]. Usually, outbreaks follow an exponential increase in the number of affected patients. It is mandatory to trace contacts with the aim to achieve early identification and screening of possible colonized patients that might be responsible for persistence of $C$. auris. Patients potentially or already colonized should be placed in single rooms with contact isolation precautions. Screening should be applied for contacts and patients previously hospitalized in healthcare settings where $C$. auris isolation was confirmed. Hand hygiene (with alcohol or chlorhexidine hand rubs), wearing of protective clothing, and skin and environmental/ equipment decontamination should be performed to prevent ongoing transmission.

\section{Global Surveillance}

Aiming to support implementation measures on global surveillance on antimicrobial resistances, in 2016, the World Health Organization [14] launched the Global Resistance Surveillance System (GLASS). The emergence of $C$. auris and progressive spread of infections caused by other resistant pathogens has strengthened the need for a surveillance network for antimicrobial resistance globally for critically ill patients' safety. 
It is hard to predict future $C$. auris diffusion. There will be outbreaks also in countries in which $C$. auris has been not reported yet? Will new MDR clones continue to emerge? Will we be able to apply effective antifungal stewardship programs and control measures? By now, global surveillance, improving knowledge, and taking care of the A.U.R.I.S. major issues may be the best ways to face $C$. auris challenge.

\section{Abbreviations}

CDC: Centers for Disease Control and Prevention; ECDC: European Center for Disease Prevention and control; GLASS: Global Resistance Surveillance System; ICU: Intensive care unit; MDR: Multidrug resistant; MIC: Minimum inhibitory concentrations

\section{Acknowledgements}

None.

Funding

None.

\section{Availability of data and materials}

Not applicable.

\section{Authors' contributions}

AC, GM, AG, MB, and DE conceived the content, wrote the manuscript, and approved the last version.

\section{Ethics approval and consent to participate}

Not applicable.

\section{Consent for publication}

Not applicable.

\section{Competing interests}

AC is a member of the Advisory Board of Critical Care. GM and DE declare to have no competing interests. AG received grants, fees for educational presentation, and advisory board membership, without any relationship to the submitted work, from Pfizer, Merck Sharp, and Gilead. Outside the submitted work, MB has received funding for scientific advisory boards, travel and speaker honoraria from Angelini, AstraZeneca, Bayer, Biomerieux, Cidara, Cubist, Gilead, Pfizer, Melinta Therapeutics, Menarini, MSD, Nabriva, Paratek, Roche, Shionogi, Tetraphase, The Medicines Company and Astellas Pharma Inc

\section{Publisher's Note}

Springer Nature remains neutral with regard to jurisdictional claims in published maps and institutional affiliations.

\section{Author details}

${ }^{1}$ Department of Surgical, Oncological and Oral Science (Di.Chir.On.S.). Section of Anesthesia, Analgesia, Intensive Care and Emergency. Policlinico Paolo Giaccone, University of Palermo, Via del vespro 129, 90127 Palermo, Italy. ${ }^{2}$ Infectious Diseases Division, Department of Medicine, University of Udine and Santa Maria della Misericordia University Hospital, Piazzale Santa Maria della Misericordia 15, Udine, Italy. ${ }^{3}$ Oxford University Hospitals NHS

Foundation Trust, Oxford OX3 9DU, UK.

Received: 29 March 2019 Accepted: 17 April 2019

Published online: 02 May 2019

\section{References}

1. Cortegiani A, Misseri G, Fasciana T, Giammanco A, Giarratano A, Chowdhary A. Epidemiology, clinical characteristics, resistance, and treatment of infections by Candida auris. J Intensive Care. 2018;6:69.

2. Osei Sekyere J. Candida auris: a systematic review and meta-analysis of current updates on an emerging multidrug-resistant pathogen. MicrobiologyOpen. 2018;7:e00578.
3. Ruiz-Gaitan A, Moret AM, Tasias-Pitarch M, Aleixandre-Lopez Al, MartinezMorel H, Calabuig E, et al. An outbreak due to Candida auris with prolonged colonisation and candidaemia in a tertiary care European hospital. Mycoses. 2018;61:498-505.

4. Cortegiani A, Misseri G, Chowdhary A. What's new on emerging resistant Candida species. Intensive Care Med. 2018. https://doi.org/10.1007/s00134018-5363-X.

5. Rhodes J, Abdolrasouli A, Farrer RA, Cuomo CA, Aanensen DM, ArmstrongJames D, et al. Genomic epidemiology of the UK outbreak of the emerging human fungal pathogen Candida auris. Emerg Microbes Infect. 2018;7:43.

6. Lockhart SR, Etienne KA, Vallabhaneni S, Farooqi J, Chowdhary A, Govender $N P$, et al. Simultaneous emergence of multidrug-resistant Candida auris on 3 continents confirmed by whole-genome sequencing and epidemiological analyses. Clin Infect Dis. 2017;64:134-40.

7. Kordalewska M, Lee A, Park S, Berrio I, Chowdhary A, Zhao Y, et al. Understanding echinocandin resistance in the emerging pathogen Candida auris. Antimicrob Agents Chemother. 2018;62:e00238-18.

8. Madder H, Moir I, Moroney R, Butcher L, Newnham R, Sunderland M, et al. Multiuse patient monitoring equipment as a risk factor for acquisition of Candida auris. bioRxiv. 2017:149054. https://doi.org/10.1101/149054.

9. Eyre DW, Sheppard AE, Madder H, Moir I, Moroney R, Quan TP, et al. A Candida auris outbreak and its control in an intensive care setting. N Engl J Med. 2018;379:1322-31.

10. Sherry L, Ramage G, Kean R, Borman A, Johnson EM, Richardson MD, et al. Biofilm-forming capability of highly virulent, multidrug-resistant Candida auris. Emerg Infect Dis. 2017;23:328-31.

11. Kean R, McKloud E, Townsend EM, Sherry L, Delaney C, Jones BL, et al. The comparative efficacy of antiseptics against Candida auris biofilms. Int J Antimicrob Agents. 2018

12. Ku TSN, Walraven CJ, Lee SA. Candida auris: disinfectants and implications for infection control. Front Microbiol. 2018;9:726.

13. https://ecdc.europa.eu/en/publications-data/rapid-risk-assessment-candidaauris-healthcare-settings-europe. Accessed 20 Mar 2019.

14. https://www.who.int/glass/en/. Accessed 20 Mar 2019 